\title{
The Time has Come for All States to Measure Racial Discrimination: A Call to Action for the Pregnancy Risk Assessment Monitoring System (PRAMS)
}

\author{
Joanna Almeida ${ }^{1}\left[\right.$ Candice Belanoff $^{2} \cdot$ Kristin F. Erbetta $^{1}$ \\ Accepted: 21 April 2021 / Published online: 4 May 2021 \\ (c) The Author(s), under exclusive licence to Springer Science+Business Media, LLC, part of Springer Nature 2021
}

\begin{abstract}
Introduction Racial/ethnic inequities in preterm birth (PTB) are well documented. Most of this research has focused on maternal behavioral and socio-demographic characteristics. However, the full magnitude of the racial/ethnic gap remains inadequately understood. Studies now point to the role of racial discrimination in producing PTB inequities, but limitations exist, namely the use of a single, dichotomous item to measures discrimination and the limited generalizability of most studies which have been conducted in single cities or states.

Methods In this commentary we briefly review extant research on explanations for racial/ethnic inequities in PTB, and the role of racial discrimination in producing the racial/ethnic gap in adverse birth outcomes such as PTB.

Results The Pregnancy Risk Assessment Monitoring System (PRAMS), a state-level, population-based survey, annually collects data from 51 states and cities ("states") on maternal behaviors and experiences in the perinatal period. The questionnaire consists of mandatory "Core" questions, and optional "Standard" questions. Currently 22 states include a "Standard" question on discrimination; 29 do not. PRAMS offers a unique opportunity to systematically assess discrimination among a diverse, population-based sample across the US.

Discussion We urge PRAMS to at least include the current measure of discrimination as a mandatory "Core" question. Ideally, PRAMS should include a validated discrimination scale as a "Core" question. The time has come to name and assess the impact of discrimination on adverse birth outcomes. PRAMS can play a vital role in helping to close the racial/ethnic gap in PTB.
\end{abstract}

Keywords Racial/ethnic inequities $\cdot$ Preterm birth $\cdot$ Racial discrimination $\cdot$ PRAMS

\section{Significance}

What is already known on this subject: Racial/ethnic inequities in preterm birth (PTB) are well documented, but explanations for these inequities remain inadequately understood. Racial discrimination likely plays a role in PTB inequities, but shortcomings of extant research include limited generalizability of most studies. The Pregnancy Risk Assessment Monitoring System (PRAMS) collects data annually from

Joanna Almeida

Joanna.almeida@simmons.edu

1 Simmons School of Social Work, Simmons University, 300 The Fenway, Office P412-B, Boston, MA 02115, USA

2 Boston University School of Public Health, Boston University, 715 Albany Street, Boston, MA 02118, USA
51 states and cities on maternal behaviors and experiences in the perinatal period, but does not systematically assess racial discrimination.

What this study adds: PRAMS offers an opportunity to assess discrimination among a diverse, population-based sample across the US. We urge PRAMS to include a discrimination measure on the questionnaire. PRAMS can play a vital role in closing the racial/ethnic gap in PTB.

\section{Adverse Birth Outcomes and Racial/Ethnic Inequities in the US}

The infant mortality rate is an important indicator of a nation's overall health (Centers for Disease Control \& Prevention, 2020a), but the United States (US) ranks poorly compared to other industrialized countries in both 
prevalence and rate of reduction over time (Singh \& Yu, 2019). Although there are many causes of infant mortality, preterm birth (PTB), defined as any birth before 37 weeks of gestation, is among the most common (Centers for Disease Control \& Prevention, 2020a). An objective of Healthy People 2020 was to reduce the overall rate of PTB in the US from $10.4 \%$ in 2007 to $9.4 \%$ in 2020, Department of Health \& Human Services, Healthy People (2020). While natality data for 2020 are not yet available, the rate of PTB actually increased from $9.6 \%$ in 2014 to $10.0 \%$ in 2018 (Martin et al., 2019), suggesting the Healthy People goal will likely prove elusive. Equally disheartening are the widening racial/ ethnic inequities in PTB in the US, which account for the largest proportion of the racial/ethnic gap in infant death (Martin et al., 2019). Racial/ethnic inequities in adverse birth outcomes, particularly in rates of PTB between nonLatinx Black and white women, have been documented for decades. In 2018, the rate of PTB among non-Latinx white women was $9.1 \%$ while among non-Latinx Blacks it was 14.1\% (Martin et al., 2019). These inequities in PTB have been impervious to extensive research and improvements in access to prenatal care (Alhusen et al., 2016), and as such few answers and levers for preventive interventions exist (Bower et al., 2018; Burris \& Hacker, 2017).

\section{Explanations for Racial/Ethnic Inequities in PTB}

To date, most research on the causes of racial/ethnic inequities in PTB has focused on differences in maternal characteristics such as medical, behavioral and sociodemographic risk factors during pregnancy (Alhusen et al., 2016; Burris \& Hacker, 2017; Lu \& Halfon, 2003). For example, differential access to prenatal care is a frequently tested explanation for racial/ethnic inequities in PTB. However, increases in access to prenatal care, including among non-Latinx Blacks (Alexander et al., 2002), have not yielded lower rates of PTB among this group, or narrowed racial/ethnic inequities in adverse birth outcomes (Lu \& Halfon, 2003). Moreover, despite poor access to prenatal care and a socioeconomic profile similar to non-Latinx Blacks, Latinx immigrants, particularly Mexican women have relatively low rates of PTB. This phenomenon by which Latinx immigrants have good birth outcomes, notwithstanding their low socioeconomic status (SES), is referred to as the "Latino Paradox" and calls into question the role of access to prenatal care, as well as SES in explaining racial/ethnic inequities in PTB (Cervantes et al., 1999).

A further thesis posits that engagement in risky behaviors (e.g. substance use) explains racial/ethnic inequities in adverse birth outcomes (Cervantes et al., 1999). Studies have nevertheless shown that Black and Latinx women are in fact less, or at least no more likely than their nonLatinx white counterparts to smoke or drink alcohol during pregnancy (Singh \& Yu, 2019). Yet another assertion of this work is that racial/ethnic differences in SES drive inequities in PTB. While SES is associated with adverse birth outcomes such as PTB (Braveman et al., 2015), and on average racial/ethnic minorities have lower SES than non-Latinx whites, even after controlling for SES, research has found residual inequities across race/ethnicity (Braveman et al., 2015).

Taken together, SES and other maternal characteristics and behaviors do not fully explain the entrenched racial/ ethnic inequities in PTB (Lu \& Halfon, 2003), and racial/ ethnic gaps remain inadequately understood (Bower et al., 2018). Absent an understanding of the social determinants of maternal risk factors, we fail to understand the fundamental causes of inequities in adverse birth outcomes and risk "blaming the victim." Information on the social determinants is notably lacking in perinatal data sets, but is key to identifying root causes of reproductive health inequities across race/ethnicity (Witt, 2018).

\section{Interpersonal Racial Discrimination/ Structural Racism and PTB}

\section{Interpersonal Racial Discrimination}

A robust area of inquiry now centers on the role of racial discrimination and racism in producing racial/ethnic gaps in adverse birth outcomes (Alhusen et al., 2016; Burris \& Hacker, 2017; Slaughter-Acey et al., 2016). Most studies in this area have found that interpersonal racial discrimination, conceived of as differential (unfair) treatment based on race (or another socially defined group), is a form of psychosocial stress (Alhusen et al., 2016; Krieger, 2014), that plays an important role in the occurrence of and racial/ ethnic inequities in PTB (Alhusen et al., 2016; Bower et al., 2018; Braveman et al., 2015; Burris \& Hacker, 2017; Slaughter-Acey et al., 2016). Using data from 11 US states and New York City, Bower et al. (2018) reported that nonLatinx Black women who felt upset by experiences of racial discrimination had a significantly higher risk of PTB relative to women who did not feel upset by these experiences. Among a state-wide sample in California, Braveman et al. (2017), found that chronic worry about racial discrimination among non-Latinx Black, but not white women, was associated with a significantly elevated risk of PTB (Braveman et al., 2015). Another study noted that non-Latinx Black women who experienced interpersonal racial discrimination in the 12 months prior to giving birth had an elevated risk 
of PTB relative to Black women who did not report such discrimination (Slaughter-Acey et al., 2016).

\section{Structural Racism}

A burgeoning body of literature suggests that structural racism, defined as "the systems-level factors related to, yet distinct from, interpersonal racism (Hardeman et al., 2016)," adversely impacts reproductive health. Studies using both traditional (e.g. residential segregation measured with Census data), and novel (e.g. historic redlining assessed through the federally sponsored Home Owners' Loan Corporation color-coded map, Black to white ratio in elected office) measures of area-level structural racism are linked to adverse birth outcomes such as PTB (Chambers et al., 2018; Krieger et al., 2020). Moreover, these and other studies have identified structural racism as a fundamental cause of racial/ethnic inequities in maternal and infant health outcomes (Chambers et al., 2018; Krieger et al., 2018). As such, racism operates at multiple levels to harm the reproductive health outcomes of racial/ethnic minorities.

\section{Limitations of Studies on Racial Discrimination and Adverse Birth Outcomes}

Although the extant literature points to the idea that racism at the individual and structural level is a determinant of the racial/ethnic gap in PTB, the evidence is limited by a number of factors. First, some studies have been conducted in a single city, state or among small, community-based samples, which limit their generalizability (Alhusen et al., 2016). To our knowledge, only one population-based study across multiple areas (11 US states as well as New York City) examined the association between interpersonal racial discrimination and PTB. However, this study, using data from the Pregnancy Risk Assessment Monitoring System (PRAMS) was limited by its use of a single, yes/no item to measure racial discrimination which was assessed with the question: "During the 12 months before your new baby was born, did you feel emotionally upset (angry, sad, or frustrated) as a result of how you were treated based on your race?" but slightly varied in wording across states (Bower et al., 2018). Moreover, the measure only inquired about racial discrimination during the 12 months prior to birth, and it has long been postulated that the effects of racial discrimination accumulate over the life course, degrading the health of racial/ethnic minority women, particularly Blacks, as they age. Given the multifaceted and chronic nature of racial discrimination, a single, dichotomous, point-in-time item cannot plausibly capture this complex on-going exposure (Bower et al., 2018; Krieger, 2012).
A further shortcoming relates to the fact that the vast majority of existing research on the relationship between racial discrimination and racial/ethnic inequities in adverse birth outcomes has largely focused on non-Latinx Blacks. Birth outcomes are undoubtedly worse for US-born nonLatinx Blacks (Martin et al., 2019), and relative to other racial/ethnic minorities, and to non-Latinx whites, Blacks disproportionately face racial discrimination and its pernicious effects on health (Lee et al., 2019). Nevertheless, there is a paucity of research on other racial/ethnic minorities and immigrants in spite of several demographic trends. In 2017, $14 \%$ of the overall US population was foreign-born, and these recent immigrants come mainly from Asia and Latin America (Vespa et al., 2020). Additionally, by 2045, non-Latinx whites will be the racial/ethnic minority in the US, driven largely by growth in population share of Latinx and Asians (Vespa et al., 2020). This gap in the literature is especially notable for three reasons. First, US-born Latinx report more discrimination than their foreign-born counterparts (Almeida et al., 2016), which might help explain the worsening birth outcomes observed with increased time in the US, and among the US-born offspring of immigrants. Second, a seminal study by Lauderdale (2006) documented that Arabic-named women had an increased risk of PTB during the 6 months following September 11, 2001 (a period of heightened discrimination towards Middle Eastern populations), relative to similar women without Arabic names who gave birth during the 6 months prior to 9/11 (Lauderdale, 2006). Third, amidst the current Covid- 19 pandemic, reports of discrimination against Asians in the US have surged (Human Rights Watch, 2020). As such, we need to extend our understanding of how racial discrimination impacts the birth outcomes of multiple racial/ethnic minority groups by assessing this construct with a validated measure among a nationally representative, population-based sample (Alhusen et al., 2016). Finally, studies demonstrating the noxious impact of area-level structural racism on birth outcomes, have not tested potential pathways such as interpersonal discrimination (Chambers et al., 2018; Krieger et al., 2020). Therefore, without systematically measuring interpersonal racial discrimination in large scale, population-based studies, its role in producing and perpetuating racial/ethnic inequities in adverse birth outcomes among multiple racial/ ethnic minorities and immigrants remains unclear.

\section{An Opportunity for National Surveillance of Interpersonal Racial Discrimination in the Perinatal Period and Beyond}

Responding to the stalled decline in infant mortality rates, the US Centers for Disease Control and Prevention (CDC) initiated the Pregnancy Risk Assessment Monitoring 
System (PRAMS) in 1987 (Centers for Disease Control \& Prevention, 2020b). PRAMS is an ongoing state-level, population-based surveillance system that collects data on various maternal behaviors and experiences before, during, and shortly after pregnancies that result in a live birth or fetal death. Through a partnership with the CDC's Division of Reproductive Health, PRAMS is conducted annually by 47 participating states as well as Washington DC, New York City, Puerto Rico, and the Great Plains Tribal Chairman's Health Board (hereafter referred to as "states"). Currently PRAMS surveillance covers $83 \%$ of all births in the US (Centers for Disease Control \& Prevention, 2020b). The main purpose of this surveillance system is to collect, analyze, and disseminate population-based data, and to support the use of these data to develop policies and programs that help reduce maternal and infant morbidity and mortality, identify why some infants are healthy and others are not, and ideally eliminate racial/ethnic inequities in reproductive health outcomes (Centers for Disease Control \& Prevention, 2020b).

PRAMS questionnaires consist of three types of questions: "Core" questions that are mandatory for all participating PRAMS states, "Standard" questions developed by the CDC and made available for selection to all participating states, and state-developed questions. In designing their survey instrument, states are allowed to choose from the library of Standard questions or develop and pilot their own questions to address state priority topics. In addition, brief question supplements can be appended to the regular PRAMS survey as a way for states to quickly collect data on emerging topics of concern. For example, in 2016, the CDC funded the Puerto Rico Department of Health to conduct a rapid assessment of Zika virus awareness and use of preventive measures (Centers for Disease Control \& Prevention, 2020b).

\section{A Call to Action-The Way Forward}

To our knowledge 22 states currently include a Standard question that assesses interpersonal racial discrimination on their PRAMS survey instrument, and a few have added their own items on this construct. However, 29 participating states do not measure racial discrimination at all. As such, the ability to monitor exposure to interpersonal racial discrimination, and study its impact on maternal and infant health including racial/ethnic inequities at the national level, remains out of reach. The PRAMS questionnaire is currently in its eighth version, and revisions take place every 3-5 years. Through the systematic collection of data on the periods before, during and shortly after delivery, PRAMS has been collecting information on a wide range of risk factors that influence maternal and infant health for over 30 years (Centers for Disease Control
$\&$ Prevention, 2020b). Previous studies using PRAMS data have noted the limitations of the dichotomous one-item discrimination Standard question currently used by 14 states, and called for the inclusion of a psychometrically sound, multi-item measure of this complex and important construct (Alhusen et al., 2016; Bower et al., 2018).

The PRAMS survey provides a unique opportunity to assess interpersonal discrimination among a racially/ethnically diverse population-based sample across the US. With the inclusion of a robust measure of interpersonal racial discrimination on the PRAMS survey, Maternal \& Child Health $(\mathrm{MCH})$ researchers will be able to test whether this factor accounts for racial/ethnic inequities in adverse birth outcomes such as PTB, which remain unexplained by maternal sociodemographic, behavioral and medical variables currently collected on the PRAMS survey. Additionally, by collecting data on interpersonal discrimination, PRAMS could help illuminate explanations for the worsening birth outcomes observed among the US-born offspring of immigrants. Furthermore, such an inclusion would enable researchers to link PRAMS data to area-level measures of structural racism to formally test interpersonal discrimination as a pathway by which structural racism is embodied and ultimately harms the reproductive health outcomes of racial/ethnic minorities (Alhusen et al., 2016; Krieger, 2014). We urge the CDC to, at a minimum, make the current Standard question on discriminations a Core question asked by all participating states. Ideally, however, we call for PRAMS to include in its Core questionnaire a validated scale of interpersonal racial discrimination to assess the frequency, duration and intensity of this deleterious exposure in multiple realms (e.g. health care, employment) over the life course (Krieger, 2012; Witt, 2018).

The time has come to name, measure and assess the impact of racial discrimination on maternal and child health, and the persistent racial/ethnic inequities. For too long, the $\mathrm{MCH}$ field has been prevented from systematically documenting and studying "the elephant in the room." It is time that $\mathrm{MCH}$ was equipped to confront the legacy and impact of racism and discrimination on the health of women and their children. PRAMS data can play a vital role in identifying fundamental causes of racial/ethnic inequities in birth outcomes. Collecting such data is the first step to developing multilevel interventions that promote maternal and child health, and ultimately close the racial/ethnic gap in adverse birth outcomes such as PTB (Witt, 2018).

Author Contributions JA conceived of and wrote the paper, CB \& KE wrote the paper.

Funding We received no funding for this manuscript. 


\section{Declarations}

Conflict of interest All authors declare that they have no conflict of interest.

\section{References}

Alexander, G., Kogan, M., \& Nabukera, S. (2002). Racial differences in prenatal care use in the United States: Are disparities decreasing? American Journal of Public Health, 92, 1970-1975.

Alhusen, J., Bower, K., Epstein, E., \& Sharps, P. (2016). Racial discrimination and adverse birth outcomes: An integrative review. Journal Midwifery \& Women's Health, 61(6), 707-720.

Almeida, J., Biello, K., Wintner, S., Pedraza, F., \& Viruell-Fuentes, E. (2016). The association between anti-immigrant policies and perceived discrimination among Latinos in the US: A multilevel analysis. Social Science \& Medicine-Population Health, 2, 897 903. https://doi.org/10.1016/j.ssmph.2016.11.003

Bower, K., Geller, R., Perrin, N., \& Alhusen, J. (2018). Experiences of racism and preterm birth: Findings from a Pregnancy Risk Assessment Monitoring System, 2004 through 2012. Women's Health Issues Journal, 28(6), 495-501.

Braveman, P., Heck, K., Egerter, S., Dominguez, T. P., Rinki, C., Marchi, K. S., \& Curtis, M. (2017). Worry about racial discrimination: A missing piece of the puzzle of Black-White disparities in preterm birth? PLOS ONE, 12(10), e0186151. https://doi.org/ 10.1371/journal.pone.0186151

Braveman, P., Heck, H., Egerter, S., Marchi, K., Parker-Dominguez, T., Cubbin, C., Fingar, K., Pearson, J. A., \& Curtis, M. (2015). The role of socioeconomic factors in black-white dispartieis in preterm birth. American Journal of Public Health, 105(4), 694-702. https://doi.org/10.2105/AJPH.2014.302008

Burris, H., \& Hacker, M. (2017). Birth outcome racial disparities: A result of intersecting social and environmental factors. Seminars in Perinatology, 41(6), 360-366. https://doi.org/10.1053/j.sempe ri.2017.07.002

Centers for Disease Prevention \& Control. (2020b). What is PRAMS? Retrived June 6, 2020, from https://www.cdc.gov/prams/index. htm

Centers for Disease Prevention \& Control. (2020a). Reproductive Health. Retrieved June 6, 2020, from https://www.cdc.gov/repro ductivehealth/maternalinfanthealth/infantmortality.htm\#about

Cervantes, A., Keith, L., \& Wyshak, G. (1999). Adverse birth outcomes among native-born and immigrant women: Replicating national evidence regarding Mexicans at the local level. Maternal \& Child Health Journal, 3(2), 99-109.

Chambers, B., Toller Erausquin, J., Tanner, A., Nichols, T., \& BrownJeffy, S. (2018). Testing the association between traditional and novel indicators of county-level structural racism and birth outcomes among black and white women. Journal of Racial and Ethnic Health Disparities, 5, 966-977. https://doi.org/10.1007/ s40615-017-0444-z

Department of Health \& Human Services, Healthy People (2020). Maternal, infant, and child health. Department of Health and Human Services. https://www.healthypeople.gov/2020/leadinghealth-indicators/2020-lhi-topics/Maternal-Infant-and-ChildHealth/data. Accessed 6 July 2020
Hardeman, R. R., Medina, E. M., \& Kozhimannil, K. B. (2016). Structural racism and supporting Black lives-The role of health professionals. The New England Journal of Medicine, 375(22), 2113-2115. https://doi.org/10.1056/NEJMp1609535

Human Rights Watch. (2020). Covid-19 fueling anti-Asian racims and xenophobia worldwide. Retrieved June 30, 2020, from https:// www.hrw.org/news/2020/05/12/covid-19-fueling-anti-asian-racism-and-xenophobia-worldwide

Krieger, N. (2012). Methods for the scientific study of discrimination and health: An ecosocial approach. American Journal of Public Health, 102, 936-945. https://doi.org/10.2105/AJPH.2011.300544

Krieger, N. (2014). Discrimination and health inequities. International Journal of Health Services, 44(4), 643-710. https://doi.org/10. 2190/HS.44.4.b

Krieger, N., Huynh, M., Li, W., \& Van Wye, G. (2018). Severe sociopolitical stressors and preterm births in New York City: 1 September 2015 to 31 August 2017. Journal of Epidemiology \& Community Health, 72(12), 1147-1152. https://doi.org/10.1136/ jech-2018-211077

Krieger, N., Van Wye, G., Huynh, M., Waterman, P., Maduro, G., Li, W., Gwynn, R. C., Barbot, O., \& Bassett, M. (2020). Structural racism, historical redlining, and risk of preterm birth in New York City, 2013-2017. American Journal of Public Health, 10(7), 1046-1053. https://doi.org/10.2105/AJPH.2020.305656

Lauderdale, D. (2006). Birth outcomes for Arabic-named women in California before and after September 11. Demography, 43(1), 185-201. https://doi.org/10.1353/dem.2006.0008

Lee, R., Perez, A., Boykin, C., \& Mendoza-Denton, R. (2019). On the prevalence of racial discrimination in the United States. PLoS ONE, 14(1), e0210698. https://doi.org/10.1371/journal.pone. 0210698

Lu, M., \& Halfon, N. (2003). Racial and ethnic disparities in birth outcomes: A life-course perspective. Maternal \& Child Health Journal, 7(1), 13-30.

Martin, J. A., Hamilton, B. E., Osterman, M. J., \& Driscoll, A. K. (2019). Births: Final data for 2018. In National vital statistics reports (Vol. 63). National Center for Health Statistics.

Singh, G., \& Yu, S. (2019). Infant mortality in the United States, 19152017: Large social inequalities have persisted for over a century. International Journal of MCH and AIDS, 8(1), 19-31.

Slaughter-Acey, J., Sealy-Jefferson, S., Helmkamp, L., Caldwell, C., Osypuk, T., Platt, R., Straughen, J. K., Dailey-Okezie, R. K., Abeysekara, P., \& Mesra, D. (2016). Racism in the form of micro aggressions and the risk of preterm birth among black women. Annals of Epidemiology, 26(1), 7-13. https://doi.org/10.1016/j. annepidem.2015.10.005

Vespa, J., Medina, L., \& Armstrong, D. M. (2020). Demographic turning points for the United States: Population projections for 2020 to 2060. Current Population Reports. Retrieved July 6, 2020, from https://www.census.gov/content/dam/Census/library/publications/ 2020/demo/p25-1144.pdf

Witt, W. (2018). The future of maternal and child health data in the United States. American Journal of Public Health, 108(10), 1277 1279. https://doi.org/10.2105/AJPH.2018.304663

Publisher's Note Springer Nature remains neutral with regard to jurisdictional claims in published maps and institutional affiliations. 